\title{
Maze orientation, visual and vestibular cues in two-maze spontaneous alternation of rats
}

\author{
M. POTEGAL \\ New York State Psychiatric Institute, New York, New York 10032 \\ and \\ M. J. DAY and L. ABRAHAM \\ Columbia University, Teachers College, New York, New York 10027
}

\begin{abstract}
In a study of the cues involved in spontaneous alternation in which the first turn took place in one maze and the second turn in a different maze, we found: (1) identical levels of significant, above-chance, alternation whether the mazes were parallel or perpendicular to each other; (2) blinding did not affect alternation rates; and (3) bilateral vestibular neurotomy produced a severe and long-lasting (up to 1 year) reduction of spontaneous alternation under all cue conditions tested. This reduction was greatest in the parallel condition. We conclude that, in the absence of visually differentiated maze arms, vision plays no role in two-maze spontaneous alternation while kinesthetic cues may play a role. Alternation depends on the integrity of the vestibular system, although the specificity of the vestibular alternation deficit remains to be determined. Data on righting reflexes in air, collected in evaluation of vestibular function, are also presented.
\end{abstract}

Douglas' (1966a) investigation of the cues for spontaneous alternation (SA) led to the claim that SA was largely controlled by spatial direction with a lesser contribution from odor trail cues. In his original study, he found that SA would occur at nearly normal rates if the rat's first turn was made in one maze and the second turn in another maze, provided both mazes were oriented in the same direction (parallel condition). If the mazes were oriented at right angles (perpendicular condition), rates fell to chance unless an odor trail was present. Douglas offered this and other evidence in support of the hypotheses that "body-turn" kinesthesis was not involved in SA but that a vestibular factor might be crucial. He further suggested, on the basis of the effects of altering visual cues, that visual input played no role in SA.

Douglas' two-maze technique would seem to be a

This work was supported in part by USPHS Grant NS 05184 to Professor Sid Gilman and by the facilities of the Motor Learning Program of Columbia University Teachers College. For their invaluable assistance in the arduous, time-consuming, and not altogether rewarding tasks of operating, maintaining, and testing subjects, we sincerely thank David Hill, Diane Jacobs, David Kornhauser, and Chung-Kit Yu. We also thank Marion Boike, Francine Castellucci, and Peter Kugler for their untiring help in innumerable pilot studies. Dr. R. Gray, of Columbia University, College of Physicians and Surgeons, prepared and analyzed the middle ear cultures. Professor S. Gilman provided steadfast support throughout this investigation; Professor Susan Manning contributed her advice. Dr. L. Abraham's current address is: Department of HPER, University of Texas, Austin, Texas 78712 . useful procedure for eliminating a variety of intramaze cues. If, indeed, SA occurs reliably with the two-maze technique despite the absence of intramaze cues, then this technique would provide a good vehicle to evaluate Douglas' claims further in rats in whom visual or vestibular inputs had been surgically eliminated. However, the replication of orientation-dependent two-maze SA has had only mixed success in the hands of other investigators. Bronstein, Dworken, Bilder, and Wolkoff (1974) and Sherrick and Dember (1966) failed to obtain significant rates of SA in a two-maze parallel situation. Lester (1968) and Still and MacMillan (1969) replicated the nonoccurrence of SA in the two-maze perpendicular condition; the latter authors (1969, 1975) reported significant SA in this situation if olfactory cues other than odor trails were present. In view of the potential methodological advantages of the two-maze procedure, as well as the theoretical questions concerning the cues involved in spatial orientation of SA, we thought it worthwhile to reexamine the characteristics of two-maze SA. If Douglas' results could be replicated in a first experiment, we could then use the powerful technique of surgical ablation to study the contribution of visual and vestibular cues to SA in subsequent experiments.

\section{EXPERIMENT 1}

\author{
Method \\ Subjects. The subjects were 39 experimentally naive male,
}


Long-Evans, $250-300 \mathrm{~g}$ hooded rats singly housed with ad-lib food and water. Since pilot studies had suggested a higher frequency of refusal to run in the perpendicular condition, 21 subjects were assigned to that condition.

Apparatus and Procedure. Two identical T-mazes were placed on sheets of Kraft 90 wrapping paper stacked on the floors of two adjoining rooms. Mazes were constructed of unpainted 9.4-mm plywood with stems and arms $40 \mathrm{~cm}$ long, $10 \mathrm{~cm}$ wide, and $37 \mathrm{~cm}$ high. In each maze, a vertical guillotine door in the stem formed a $25-\mathrm{cm}$-long start compartment. A horizontal sliding door was placed at the entrance to each arm. The only illumination was provided by a $7.5-\mathrm{W}$ bulb located above the choice point. The paper floor of each maze was changed for each rat by removing the top sheet of the stack under the maze. In the parallel condition, the stem of the second maze was aligned with the stem of the first maze and faced the same direction. In the perpendicular condition, the arms of the second maze were oriented along the axis of the stem of the first maze. Each maze and room was used for the first trial on alternate days. On any given day, the first trial of each trial-pair in the parallel and perpendicular conditions was run in the same maze, in the same position, in the same room; the second trial differed only in maze orientation.

To reduce fearfulness in the maze situation, a group of 3-5 rats was habituated in a maze, with all doors open, for $20 \mathrm{~min}$ on 3 days prior to testing. To begin a trial, the rat was placed in the start compartment. Ten seconds later, the vertical door in the stem was raised and timing with a stopwatch began. When the subject left the start compartment, the vertical door was lowered to prevent reentry. When his whole body had entered one of the arms, the door to that arm was closed and the elapsed time (latency) and left or right choice were recorded. He was confined for $45 \mathrm{sec}$ in the chosen arm and then was removed from the first maze and carried into the adjoining room where he was placed in the delay chamber for a few seconds. He was then placed in the second maze to begin the second trial. The entire intertrial interval was approximately $15 \mathrm{sec}$. To minimize the amount of intertrial vestibular stimulation, the rat was lifted from the first maze and carried from room to room without disturbing his posture or orientation. As the rat was lowered into the start compartment for his second trial, he was gently rotated from his carrying position through the smallest angle necessary to orient him parallel to the stem and facing the choice point. Alternation was scored when the rat entered the arm opposite to that entered on the immediately preceding trial. If the rat entered the arm chosen previously, a nonalternation was scored. If the latency exceeded 4 min (balk time limit), the animal was removed from the maze and received no further testing that day. The subjects were given one trial pair each day until they had completed 10 trial pairs; rats which balked on three consecutive occasions and could not be induced to run by rehabituation to the mazes were dropped from the study.

Data analysis. Since the chance level of expected alternations is a function of preexisting turning tendencies, raw alternation score comparisons between groups can be misleading. In each case, we have therefore reported Manning's (1973) adjusted alternation rate (AAR)' first, followed by the raw alternation percentage in parentheses. The AAR index varies from -1.0 (maximum perseveration) to +1.0 (maximum alternation), with a score of 0.0 indicating a chance level of alternation. All statistical tests of alternation have been performed on the AAR scores.

Dember, Sherrick, and Harris (1966) reported that, in the perpendicular condition, a rat may choose the arm on Trial 2 which is opposite to stem orientation on Trial 1. If this is true, an increased proportion of right turns would be expected on Trial 2 when Maze 2 has been rotated $90^{\circ}$ clockwise to Maze 1 . Left-turn proportions should increase on Trial 2 when Maze 2 has been rotated counterclockwise. These proportions were calculated.

\section{Results}

Balk rates were higher in the perpendicular $(33 \%)$ than in the parallel $(6 \%)$ condition; the final groups contained 14 and 17 subjects, respectively. Mean AARs were $.34(66 \%)$ for the parallel condition and $.31(66 \%)$ for the perpendicular condition. Scores for both groups were significantly different from 0.0 (parallel: $\mathrm{t}=4.48, \mathrm{df}=16, \mathrm{p}<.001$; perpendicular: $\mathrm{t}=2.90$, $\mathrm{df}=13, \mathrm{p}<.02$ ) and were not different from each other $(t=.28)$. The variance of the perpendicular scores $(0.15)$ was somewhat greater than that of the parallel scores $(0.09)$. There were no differences in latencies on first or second trials, between or within groups, or as a function of alternation or nonalternation. Contrary to the finding of Dember et al. (1966), there was no indication of Trial 1 stem orientation effects on Trial 2 turns in the perpendicular condition.

\section{Discussion}

We have confirmed Douglas' (1966a) report of significant SA in the two-maze parallel condition. We have no clear explanation for the failures of Bronstein et al. (1974) and Sherrick and Dember (1966) to replicate Douglas' results.

We also found significant SA in the perpendicular condition, although Douglas (1966a), Lester (1968), and Still and MacMillan (1969) did not. Lester's experiment involved: (1) food deprived rats which had had (2) prior alternation experience and which (3) were rewarded with food in the arms. All three conditions tend to reduce SA rates (Richman, Juels, Peckinpaugh, \& Stutz, 1969). Thus, Lester's replication of Douglas' work would have been methodologically valid only if he also had demonstrated that similarly treated rats would still alternate in the twomaze parallel situation. Still and MacMillan's (1969) replication was less ambiguous, but, as these authors themselves noted, while SA rates in their perpendicular condition were not different from chance, they also were not different from significant abovechance rates in the other conditions of their experiment. In our study, the variance of the scores and the tendency of rats to balk was somewhat greater in the perpendicular than in the parallel condition; perhaps these effects reflect the operation of factors which produced nonalternation in the perpendicular condition of other studies. At the current time, we can only conclude that all the factors determining the rates of SA have yet to be identified.

In terms of Douglas' scheme of vestibular guidance of SA, our results bear either of two interpretations: (1) Kinesthetic (body turn) cues are sufficient to maintain SA in the absence of intramaze cues, or (2) vestibular information may be used only to record a particular turn and other mechanisms are 
used by the rat to determine maze orientation (Still \& MacMillan, 1969). Since subjects could see while being carried between mazes, it was necessary to rule out any visual contribution to SA in this situation before going on to study these possibilities.

\section{EXPERIMENT 2}

Douglas' (1966a) conclusion that visual input is not necessary for SA is supported by the finding that blinded rats show no SA deficits (Dember, 1958; Dember \& Roberts, 1958; Klein \& Brown, 1969). In these single maze experiments on blind animals, however, additional intramaze cues (e.g., olfaction) were available. Furthermore, studies subsequent to Douglas' report have found SA to be affected by visual cue manipulation (see O'Connell, 1971, for a review). In view of the contradictions in the literature, we decided to evaluate the effects of blinding on two-maze SA in which intramaze cues are eliminated. In addition, we tested the same blinded and control subjects in a second condition in which olfactory and other intramaze cues were present.

\begin{abstract}
Method
Subjects and Surgery. Subjects, selected and maintained as in Experiment 1, were anesthetized with methoxyflurane (Penthrane), the eyeballs excised with fine scissors, and the orbits filled with Bacitracin antibiotic ointment. Sham-operated controls were only anesthetized; all subjects were allowed 4 days to recover before being habituated to the mazes. Nineteen blinded and 13 shamoperated rats completed testing.

Apparatus and Procedure. All subjects were given five trial pairs under the two-maze parallel condition of Experiment 1. Upon completion of these trial pairs, they were then given an additional trial pair/day in a single maze for 5 days (intramaze cue condition). After Trial 1 of each of these latter trial pairs, the rat was removed from the arm and placed in a delay chamber while the maze and paper floor beneath it were rotated $90^{\circ}$ to the Trial 2 position. The rat was then placed in the maze stem to begin the second trial. Starting position and direction of rotation of the maze were varied from day to day. A fresh paper floor was used for each subject. The intramaze cue condition thus duplicated the perpendicular condition of Experiment 1 with the addition of intramaze cues.
\end{abstract}

\section{Results and Discussion}

In the parallel condition, blind animals produced an AAR of $.32(60 \%)$, which was greater than 0.0 at $\mathrm{p}<.01(\mathrm{t}=3.52, \mathrm{df}=18)$. Sham-operated rats' AAR was $.25(60 \%)$, which was not significantly different from that of the blind group $(t=0.41)$. Though sham AARs were also not significantly different from zero $(\mathrm{t}=1.99, .05<\mathrm{p}<.1$, df $=$ $12)$, it is unlikely that blinding increases SA; had the sham's $\mathrm{N}$ been as large as that of the blind group, their score would have presumably reached significance. These rates were comparable to those of Experiment 1. Also, as in Experiment 1, there were no differences in latencies as a function of trial, group, or alternation and nonalternation. In the absence of distinctively different visual stimuli in the maze arms, it appears that visual input plays no role in two-maze $\mathrm{SA}$.

In the intramaze cue condition, blind rats' AAR scores were $.31(64 \%)$; sham-operated scores were $.32(61.5 \%)$. Once again, there was no indication that SA was influenced by Trial 1 stem orientation. No difference could be found between sham-operate scores in the intramaze condition and the scores of normal rats in the perpendicular condition of Experiment 1. Since odor trail and intramaze cues were present in the former but not the latter case, the addition of these cues does not seem to have a significant effect on SA rates under the conditions of a $90^{\circ}$ intertrial maze rotation, in contrast to Douglas' (1966a) finding.

\section{EXPERIMENT 3}

A major piece of evidence supporting the hypothesis that vestibular cues are important in SA has been the demonstration that rats displaying signs of otitis-induced unilateral vestibular dysfunction have reduced SA rates (Douglas, 1966b; Franken $\&$ Baker, 1969). The simplest interpretation of these observations is that subjects persevered in choosing one arm of the maze because of their forced circling movement toward their impaired side. Although Douglas reported that his subjects' performance was random rather than perseverative, Franken and Baker's (1969) data revealed a clear perseverative trend. Tests of a preparation with symmetrical, bilateral vestibular dysfunction would be clearly preferable. Such a preparation has been developed for the rat (Potegal, Abraham, Gilman, \& Copack, 1975). This preparation leaves vision intact, but since we found in Experiment 2 that vision is not a factor in SA under our conditions, visual compensation for vestibular dysfunction was not thought to be a problem. We therefore decided to use this preparation to study vestibular contributions to two-maze parallel SA. Central compensation for vestibular damage may take place over a long period (Schaefer \& Mayer, 1974); subjects were therefore tested repeatedly over many months. To determine the specificity of any deficits obtained in the two-maze parallel condition, all subjects were also tested in two other conditions containing a variety of cues. Cinematographically recorded righting reflexes were evaluated to determine degree of vestibular dysfunction (Altman \& Sudarshan, 1975; Härd \& Larsson, 1975).

\footnotetext{
Method

Subject and Surgery. Vestibular neurotomy, involving removal of tympanic membrane and ossicles and cauterization of the vestibular nerves through the oval window (Potegal et al., 1975), was performed in 13 Long-Evans hooded rats (vestibular sub-
} 
jects). To produce a nerve lesion control group, the same general procedure was followed up to and including removal of the tympanic membrane and ossicles from 12 rats. The VII (facial) nerve was then transected at its exit from the bulla, approximately $200 \mathrm{mg}$ tetracycline were placed in the middle ear, and the wound was closed. A sham-operated control group of 16 rats was submitted to a simple exposure of the bulla followed by wound closure. The preoperative weight of all subjects was 220-550 g. Postoperatively, the animals were maintained on wet Purina rat mash until they could maintain their body weight on pellets.

Procedure. Testing began no sooner than 6 weeks postoperatively. All subjects were tested under several cue conditions in the following order: (1) parallel SA, 5 trials; (2) intramaze cue SA, 10 trials; (3) parallel SA, 5 trials; (4) multicue SA, 10 trials; (5) parallel SA, 5 trials. The final parallel condition tests were given 26-50 weeks postoperatively. The general procedure was that of Experiments 1 and 2. The multicue condition, which provided a maximum number of cues for alternation, was a onemaze task with no floor change or maze rotation between Trials 1 and 2 . Inserts in the maze arms (black and white vertical stripes vs. white) provided a visual differentiation, while differential tactile cues were provided by using floors of rough and smooth Masonite, respectively. Additional olfactory cues were provided by the odor of an anise soaked gauze pad at the end of one arm.

To evaluate vestibular function following completion of SA testing, the subjects were held ventral surface up and released from a height of $0.5 \mathrm{~m}$; their righting movements were recorded on high-speed film ( 64 frames/sec). Although there have been several reported film analyses of righting reflexes in rat (Altman \& Sudarshan, 1975; Härd \& Larsson, 1975), there has been no presentation of quantitative data. We found that, in our test conditions, head attitude at the moment of landing (first contact of a body part other than tail with the substrate) can be estimated with the aid of a Vanguard motion analyzer and is a sensitive measure of vestibular function. The results of 2 drops/subject were averaged.

\section{Results}

Neurological observations. Immediately postoperatively, all vestibular subjects showed the wellknown clinical signs of vestibular damage (Potegal et al., 1975). Though some of these signs (e.g., retropulsion) diminished or disappeared in time, a number persisted. Head nystagmus was the most obvious of the latter and could be exacerbated by "excitement" (e.g., being handled) until sacrifice. Facial subjects showed none of these signs but suffered extensive to complete loss of vibrissae movement, as expected. Substantial postoperative weight loss was seen in vestibular and facial subjects; by 26-32 weeks postoperatively, nine shamoperated animals had gained a mean of $154 \mathrm{~g}$; eight vestibular subjects had regained only $11 \mathrm{~g}$ over their preoperative weight, while five facial subjects had lost $5 \mathrm{~g}$ from their preoperative weight $(\mathrm{F}=12.21$, $\mathrm{df}=2,19, \mathrm{p}<.01)$. The mean preoperative weight of these rats was $350 \mathrm{~g}$.

The distributions of head attitude at landing in the righting reflex test are shown in Figure 1. Most sham-operated rats landed with their noses pointed down $\left(-45^{\circ}<\right.$ pitch $\left.<0^{\circ}\right)$ and little or no lateral tilt (roll $<45^{\circ}$ ), while most vestibular rats landed with noses pointed up $\left(0^{\circ}<\right.$ pitch $\left.<45^{\circ}\right)$ and considerable tilt (roll $>45^{\circ}$ ). The roll distribution of the facial group was like that of the sham group, while their pitch distribution was unexpectedly similar to that of the vestibular group. To perform chi-square analysis of these data, it was necessary to partition the groups so that an $\mathrm{N}$ of 5 could be expected in each cell. This was done by segregating scores above and below the median of each distribution. Chi square was then 13.6 for the pitch distributions and 20.6 for the roll distributions (for each test, $\mathrm{df}=2$, $\mathrm{p}<.001)$. Both roll and pitch distributions of vestibular neurotomized subjects were different from those of the sham-operated controls. The vestibular dysfunction thus indicated is consistent with our failure to identify any remnants of the vestibular nerves on postmortem microscopic examination of ears of the vestibular subjects. It should be noted that the visualization of the vestibular nerves was made difficult by the proliferation of fibrous tissue in the ear. This proliferation of tissue may have followed the development of otitis which was evident in some animals.

That the facial roll distribution was identical to that of the shams while the facial pitch distribution was similar to that of the vestibular group suggests a partial vestibular dysfunction in the facial group. Apparently, the maintenance of pitch is more susceptible to disturbance than the maintenance of roll. While weight losses of the facial and vestibular groups was similar, there were no gross clinical signs
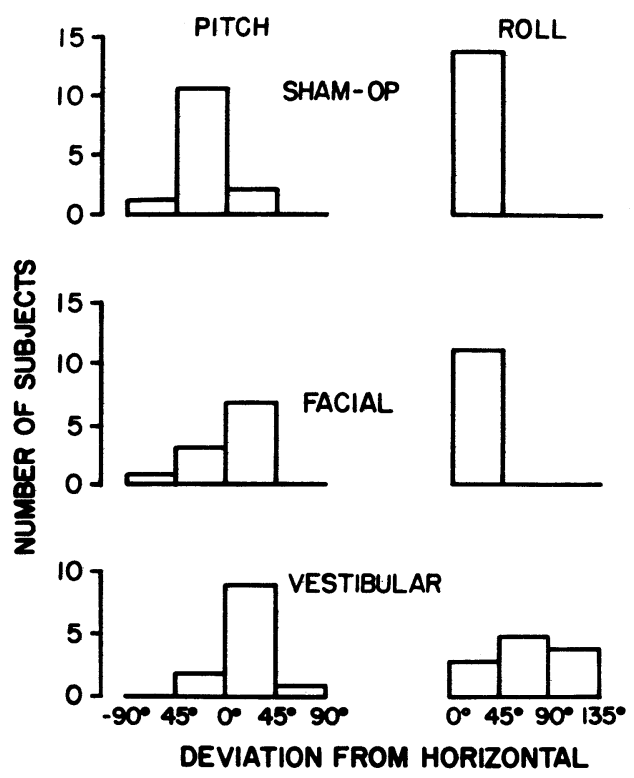

Figure 1. Estimated attitude (pitch and roll) of head at landing of 13 sham-operated, 11 facial, and 12 vestibular rats. Pitch is the angle between the long axis of the head and the plane perpendicular to the gravitational vertical. Pitch is negative if the long axis is below the plane and positive if it is above the plane. Roll is the angle, left or right, between this plane and the interaural axis. 
of vestibular dysfunction in the facial group. Thus the vestibular dysfunction of the facial group appears to be intermediate between that of the other two groups. At autopsy, facial subjects were discovered to have severe pyogenic otitis in and around the middle ear. Cultures of this material revealed diplococci, suggesting the upper respiratory tract via the Eustachian canal as source and route of infection. It is unclear whether this group's reflex dysfunction was induced by the high concentration of topically applied tetracycline, which is a nonoto-toxic agent when systematically applied, or by the subsequent infection which the drug was unsuccessful in preventing, or both. The ear infections in the facial animals appeared to be somewhat more frequent and extensive than in the vestibular subjects. In the shamoperated subjects, by contrast, only 1 ear in 10 showed signs of otitis on post mortem examination.

Behavioral observations. At different stages of testing, a number of subjects, particularly in the sham-operated group, began to balk consistently and had to be dropped from the study. By the last test occasion, 11 of 16 sham, 3 of 13 vestibular, and 2 of 12 facial subjects had stopped running; this distribution of balks is significantly different from chance $\left(\chi^{2}=9.85, \mathrm{df}=2, \mathrm{p}<.01\right)$. However, for all subjects within a group completing a given condition, $t$ tests indicated no significant differences between those subjects which dropped out at a later stage and those which eventually completed testing under all conditions.

An ANOVA was performed using the mean AAR scores for each rat that completed running in all test conditions. Fifteen trial pairs were averaged under the parallel condition, and 10 trial pairs were averaged under the intramaze and multicue conditions, respectively (see Table 1). There was a significant main effect $(\mathrm{F}=3.45, \mathrm{df}=2,22 ; \mathrm{p}<.05)$, indicating differences among surgical groups over all alternation conditions. There were no differences within groups among conditions, but there was an interaction $(F=61$, df $=4,44, p<.01)$ between groups and conditions. Post hoc Scheffé contrasts indicated that one source of this interaction is a significant difference between facial and vestibular animals within the parallel condition in the absence of differences in either of the other two nonspatial conditions. Similarly, facial animals were different from shams in the parallel condition but not in either of the other two conditions.

AAR scores for sham animals were significantly greater than 0.0 (i.e., 0.0 lay below the $95 \%$ confidence limits) for every condition; vestibular rats' scores were not different from 0.0 in any condition (see Table 1). There were no significant differences in AAR scores among the three occasions of parallel condition testing within any group (Figure 2). For the vestibular group particularly, this indicates an absence of any significant compensation for SA-
Table 1

Mean AAR and Percent Alternation Scores of Subjects Which Completed All Tests

\begin{tabular}{|c|c|c|c|c|c|c|c|}
\hline \multirow[b]{3}{*}{ Group } & \multirow[b]{3}{*}{$\mathrm{N}$} & \multicolumn{6}{|c|}{ Alternation Condition } \\
\hline & & \multicolumn{2}{|c|}{ Parallel } & \multicolumn{2}{|c|}{ Intramaze } & \multicolumn{2}{|c|}{ Multicue } \\
\hline & & AAR & $\%$ & AAR & $\%$ & AAR & $\%$ \\
\hline Sham & 5 & .60 & (80) & .67 & (82) & .87 & $(92)$ \\
\hline Facial & 10 & .35 & (67) & .35 & (68) & .46 & (72) \\
\hline Vestibular & 10 & -.04 & (46) & .07 & (49) & .24 & (60) \\
\hline
\end{tabular}

related damage up to 50 weeks postoperatively. Once again, there were no latency differences as a function of group, condition, trial, or occurrence of alternation.

\section{Discussion}

Righting reflex evaluations revealed that sham, facial, and vestibular groups were arranged in order of decreasing vestibular function, in keeping with the decreasing overall SA rates exhibited by these groups. In the group with bilateral vestibular neurotomies, SA was permanently reduced in the parallel, intramaze, and multicue conditions. In any given condition, therefore, some vestibular contributions to SA may exist independent of the type or number of maze cues available in that condition. In the parallel condition particularly, the ANOVA interaction effect indicates that facial animals were significantly impaired relative to the shams, and the vestibular animals were significantly more impaired than the facials, implying that the vestibular contribution to SA is particularly important in the absence of other cues.

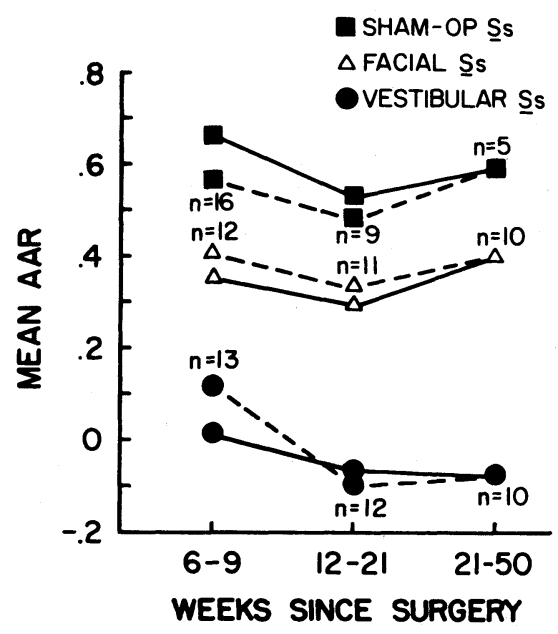

Figure 2. AAR scores for the two-maze parallel condition on the three occasions of testing. The solid line connects the mean scores of just those subjects in each group who completed all three sessions. The broken line connects the mean score on each testing occasion of all subjects (Ns given on figure) who completed testing on that occasion. 
These results are suggestive, but the specificity of the vestibular deficit in the parallel condition remains to be unequivocally demonstrated. Although the latencies of the vestibular group were not different from that of the other groups, the persistence of head nystagmus, loss of vestibulo-ocular stabilization reflex, and other sensorimotor sequelae of vestibular neurotomy may account for this group's SA deficit. In view of the numerous and severe sensorimotor consequences, we suggest that the nonspecific destruction of vestibular input is too crude a tool for analysis of SA. Perhaps selective elimination of the lateral semicircular canals would yield clearer results. Postoperative otitis remains a serious problem in the preparation of control and experimental animals; Crowley's (1972) comments on the prophylaxis of otitis in rats might be a useful guideline on the maintenance of operated subjects.

\section{GENERAL DISCUSSION}

In each of three experiments, a group of normal or sham-operated rats were given their first five SA trial pairs in the two-maze parallel condition; in each case, significant SA was found in this condition. A one-way ANOVA revealed no significant differences among these corresponding groups of Experiments 1-3. The reliable occurrence of two-maze $\mathrm{SA}$ is in accord with previous reports (e.g., Douglas, 1966a). We are unable to identify the reasons for the nonalternation in this condition reported by Bronstein et al., (1974) and Sherrick and Dember (1966).

In contrast to earlier reports, our first experiment showed that two-maze SA occurred independently of the relative orientation of the mazes. We also found that the presence of intramaze cues made no difference in alternation rates in the two-maze perpendicular condition. Experiment 2 demonstrated that blinding has no effect on SA in the two-maze parallel condition, as in other conditions employed by Dember (1958), Dember and Roberts (1958), and Klein and Brown (1969). We also failed, in both of these experiments, to show the stem orientation or latency effects of Dember et al. (1966) and Sherrick and Dember (1966). These contradictions among the present and previous reports suggests the existence of as yet unidentified variables influencing SA.

Experiment 3 supports the conclusions of Douglas (1966b) and Franken and Baker (1969) that vestibular dysfunction reduces SA. It is possible that the reduction of SA in all of the testing conditions of Experiment 3 reflects a pervasive contribution of vestibular information to the spatial guidance of SA. However, Still and MacMillan (1969) suggested that vestibular input may be only one component in a complex system for the sensory guidance of SA. Perhaps, when no other cues are available, as in our parallel condition, the contribution of the vestibular system becomes particularly important for SA. This hypothesis is consistent with the interaction effects of Experiment 3, in which statistically significant differences between shams and facials and between facials and vestibular animals were found only in the parallel condition. Alternatively, the vestibular deficits of Experiment 3 may be viewed not as related specifically to spatial orientation but as having quite nonspecific, sensorimotor effects. While this interpretation cannot be dismissed at this time, it does not appear entirely consistent with either the interaction effects or the unchanged latencies of the vestibular neurotomized subjects.

In terms of the general issues of sensory guidance of SA, the existence of significant SA in the perpendicular condition (Experiment 1) reintroduces the possibility that at least some rats may use body turn kinesthesis. Visual and other intramaze cues appear to be unneccessary for SA (Experiments 1 and 2). The evidence supporting the hypothesis of a vestibular contribution to SA (Experiment 3) or other forms of spatial orientation remains suggestive but incomplete at this time.

\section{REFERENCES}

Altman, J., \& Sudarshan, K. Postnatal development of locomotion in the laboratory rat. Animal Behaviour, 1975, 23, 896-920.

Bronstein, P. M., Dworkin, T., Bilder, B. H., \& Wolkoff, D. F. Repeated failures in reducing rat's spontaneous alternation through the intertrial disruption of spatial orientation. Animal Learning \& Behavior, 1974, 2. 207-209.

CROWLEY, D. E. Comments on otitis media in rats. Journal of Auditory Research, 1972, 12, 125-128.

DEMBER, W. N. Stimulus alternation in peripherally blinded rats. Canadian Journal of Psychology, 1958, 12, 219-221.

Dember, W. N., \& Roberts, W. Alternation behavior in peripherally blinded rats. Perceptual and Motor Skills, 1958, 8. $91-94$.

Dember, W. N., Sherrick, M. F., \& Harris, R. P., JR. Trial-two goal arm alternation to orientation of trial-one starting stem. Psychonomic Science, 1966, 6, 31-32.

Douglas, R. J. Cues for spontaneous alternation. Journal of Comparative and Physiological Psychology, 1966, 62, 171-183. (a)

Douglas, R. J. Spontaneous alternation and middle-ear disease. Psychonomic Science, 1966, 4, 243-244. (b)

Franken, R. E., \& BaKer, J. G. The effects of drive level on cues utilized in spontaneous alternation. Psychonomic Science, 1969, 16, 239-240.

HARD. E., \& LARSSON. K. Development of air righting in rats. Brain. Behavior and Evolution, 1975, 11, 53-59.

KLEIN. D., \& BRown, T. S. Exploratory behavior and spontaneous alternation in blind and anosmic rats. Journal of Comparative and Physiological Psychology, 1969, 68, 107-110.

LESTER, D. Response alternation by rats in mazes. Journal of Psychology, 1968, 70, 51-53.

Manning, S. A measure of spontaneous alternation. Behavior Research Methods \& Instrumentation, 1973, 5, 501-502.

O'Connell, R. Spontaneous alternation of brightness? Psychonomic Science, 1971, 22, 273-274. 
Potegal, M.. Abraham. L., Gilman, S., \& Copack, P. Technique for vestibular neurotomy in the rat. Physiology and Behavior. 1975. 14. 217-221.

Richman. C. L.. Juels, P.. Peckinpaugh, P.. \& Stutz, R. Olfactory cues and spontaneous alternation behavior. Psychonomic Science. 1969. 15. 23-24.

SChaEfer. K. P.. \& MAYer. D. L. Compensation of vestibular lesions. In H. H. Kornhuber (Ed.), Handbook of sensory physiology (Vot. V1/2). Vestibular system. Berlin: SpringerVerlag. 1974

Sherrick. M. F.. d Dember, W. N. The tendency to alternate direction of movement as reflected in starting stem running speed. Psichonomic Science, 1960. 6, 29-30.

SIEGEL. S. Nonparametric statistics for the behavioral sciences. New York: McGraw-Hill. 1956.

Still. A.. \& MacMillan, A. Odor trail and spontaneous alternation. Psichonomic Science, 1969, 16, 160-161.

Still. A.. \& MacMillan, A. Location by odor and turn selection as two stages in spontaneous alternation of rats. Animal Bchaviour. 1975, 23, 447-449.

\section{NOTE}

1. The AAR is calculated from the following formula (Manning, 1973, p. 502, Equation 1):

$$
A A R=\frac{O A-N(E A)}{N-N(E A)},
$$

where $\mathrm{OA}=$ number of observed alterations, $\mathrm{N}=$ number of trial pairs, and $\mathbf{E A}=$ expected number of alternations. $\mathrm{EA}$ is calculated from the formula (Manning, 1973, p. 502, Equation 3):

$$
E A=2(k / N)(1-k / N)(N / N-1),
$$

where $\mathrm{k}=$ number of right turns in the first trial of each of the $\mathrm{N}$ trial pairs.

(Received for publication January 28, 1977; revision accepted July 29,1977 .) 